\title{
Wir brauchen einen Direktstudiengang Psychotherapie - Kontra
}

\author{
We Need a New Profession of Psychotherapists - Contra
}

Kontra

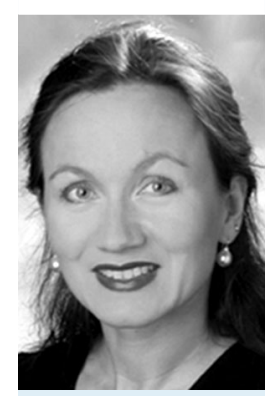

Christa RothSackenheim
Die geplante Direktausbildung zum Psychotherapeuten ist hauptsächlich deshalb ein fataler Irrweg, weil sie die längst überwunden geglaubte Trennung von Soma und Psyche institutionell zementieren würde. Es gibt zudem eine Vielzahl von Veränderungen, die dadurch angestoßen würden, die möglicherweise noch nicht ausreichend durchdacht wurden. Die Versorgung von Menschen mit psychischen Störungen darf jedoch nicht durch Kollateralschäden bedroht werden.

- Die Menschen stünden dann vor einer noch größeren Vielzahl von Berufsbezeichnungen im Psychobereich als bisher, deren Inhalt nicht transparent ist.

- Ein Abiturient müsste sich bereits vor Beginn des Studiums entscheiden, ob er ein Medizin- oder ein Psychotherapiestudium anstrebt. Es ist wohl vorhersehbar, dass sich künftig weniger junge Menschen für das Medizinstudium mit anschließender Facharztweiterbildung entscheiden werden, wenn es einen kürzeren Studiengang gibt, der möglicherweise zu ähnlichen Befugnissen mit geringerer Versorgungsverantwortung führt (z.B. keine Notdienste, keine Notfallsprechstunde, keine klinische Ausbildung mit Nachtdiensten). Dazu muss man kein Prophet sein.

- Das ordnungspolitische Ziel des BMG der Parallelisierung der Ausbildung zum Psychotherapeuten mit dem Medizinstudium würde hiermit nur formal, aber keinesfalls inhaltlich realisiert. Es würde ein neuer Studiengang mit willkürlich für psychotherapierelevant gehaltenen Inhalten geschaffen, in den ärztliche Teilkompetenzen integriert werden sollen.
- Dem so ausgebildeten Psychotherapeuten sollen bereits im Studium umfassende psychotherapeutische Kenntnisse vermittelt werden. Es fehlen jedoch dabei die entsprechenden somatischen Kenntnisse. Die Kompetenz in der somatischen Medizin ist jedoch unteilbar.

- Insofern könnten in einem solchen Direktstudiengang auch keine Grundlagen für eine Befugniserweiterung erworben werden. Psychische und hirnorganische Erkrankungen sind differenzialdiagnostisch und -therapeutisch auf das engste mit möglichen Fehlfunktionen des übrigen Körpers und Krankheiten anderer Körperorgane verbunden. Die Konzentration der Direktausbildung zum Psychotherapeuten auf die psychischen Funktionen des Gehirns blendet notwendiges ärztliches Wissen und Erfahrungen über Funktionsweise und Erkrankungen des gesamten „Systems Mensch“ aus.

- Aus dem oben Genannten ergibt sich, dass der direkt ausgebildete Psychotherapeut auch nicht einzelne bisher ärztliche und speziell bei psychischen Erkrankungen erforderliche Maßnahmen ausführen und die notwendigen Berechtigungen erhalten könnte. Dies beträfe beispielsweise das Rezeptieren von Psychopharmaka, die stationäre Einweisung in psychiatrische bzw. psychosomatische Kliniken oder die Feststellung von Arbeitsunfähigkeit aus psychischen Gründen. Derartige Berechtigungen direkt ausgebildeter Psychotherapeuten würden zu einer systematischen potenziellen Gefährdung bzw. Ausblendung komorbider Erkrankungen der ihnen anvertrauten Patienten führen.

- Wenn der neue Psychotherapeut wie angestrebt einen Facharztstatus bekäme, könnte er auch Kliniken und Abteilungen leiten. Damit würde es eine Konkurrenz zu ärztlich geleiteten Kliniken geben. Die psychotherapeutischen Kliniken würden die umfassende
Behandlung psychischer Störungen, zu denen die somatische Seite immer unteilbar dazugehört, fachlich nicht leisten können.

- Der neue Psychotherapeut würde dann Fachärzte für Psychiatrie und Psychotherapie oder andere Fachärzte anstellen und/oder ausschließlich mit der Aufgabe der Psychopharmakotherapie beauftragen können und diesen möglicherweise weisungsbefugt sein. Das ist komplett abzulehnen, denn die Verantwortung für die umfassende Behandlung von Menschen mit psychischen Erkrankungen geht nur im Einbezug der somatischen Komorbiditäten und ist eine originär ärztliche Aufgabe.

- Wenn dieser neue Heilberuf „auf den Markt" kommt, dann bedeutet dies, dass hierdurch eine Trennung von Soma und Psyche für die Zukunft unumstößlich institutionalisiert wird. Die ärztliche Versorgung von Menschen mit psychischen Störungen würde künftig komplett aus der Medizin herausgelöst. Das kann nicht im Sinne einer guten Patientenversorgung sein! Dass aus Sicht von uns Fachärzten für Psychiatrie und Psychotherapie die Direktausbildung Bedenken und sogar Ängste vor Verdrängung des eigenen Berufsstandes hervorruft, mag nicht verwundern. Doch es gibt auch zunehmenden Widerstand aus den Reihen der Psychologen, der psychologischen Lehrstuhlinhaber sowie der Leiter von Ausbildungsinstituten. Diese sagen unisono: „wir wollen kein ,Arzt light' werden, wir wollen ,richtige Psychologen` bleiben“. Es ist weiterhin auch von der Ärzteschaft unbestritten, dass einige handwerkliche Fehler des Psychotherapeutengesetzes nachgebessert werden müssen. Die Benachteiligung und Ausbeutung von Psychotherapeuten in Ausbildung (PiA) an Kliniken muss aufhören. Die Ausbildung muss inhaltlich einheitlicher werden.

Aber braucht man für diese Änderungswünsche einen komplett neuen Studiengang? Soll neben der Medizin eine formal 
komplett spiegelbildlich aufgebaute psychotherapeutische Heilkunde entstehen? Was ist der Grund dafür?

Die Kultusministerkonferenz sähe sich vor der Aufgabe, bundeseinheitliche neue Studiengänge für Psychotherapie an den Universitäten einzurichten. Das wäre eine andere Herausforderung als bisher, wo im Rahmen des Bologna-Prozesses neue Studiengänge an jeder Universität individuell entstehen können.

Es beginnt in der Antizipation des Direktstudiengangs bereits der Kampf der Fakultäten, ob der neue Studiengang an der philosophischen, der medizinischen oder sozialwissenschaftlichen Fakultät angesiedelt werden soll. Einige Einrichtungen bieten bereits Modellstudiengänge an, was aber derzeit eher der weiteren Zersplitterung Vorschub leistet.

Wenn man eigentlich im Grunde eine andere Medizin will, eine mehr sprechende, weniger technisierte Medizin, dann muss man im Medizinstudium und in den Fach- arztweiterbildungen aller Gebiete ansetzen. Die Probleme der handwerklichen Fehler des Psychotherapeutengesetzes könnten wesentlich niedrigschwelliger, kostengünstiger und sowohl für die ärztliche als auch für die psychologische Seite weniger fachidentitäts-irritierend gelöst werden.

Warum nicht für Psychologen, Pädagogen und Sozialwissenschaftler nach dem Bachelor einen einheitlichen psychotherapeutischen Zweig schaffen, in dem bis zum Master-Abschluss bereits psychotherapeutisches Grundwissen vermittelt wird? So könnten alle diese Studierenden der genannten Fächer, wenn sie sich für eine Psychotherapietätigkeit interessieren, auf ein in etwa gleiches Wissensniveau vor Beginn der postgradualen Psychotherapeutenausbildung gebracht werden. Die Weichenstellung in Richtung eines Heilberufs würde so früher als bisher erfolgen, nicht erst mit Abschluss des Psychologiestudiums.
Und last but not least: Wir würden uns einen weiteren „deutschen Sonderweg“ ersparen. Warum sollten wir uns hier anders aufstellen als alle anderen Länder weltweit?

Sie haben eine eigene Meinung zu diesem

Thema? Dann schreiben Sie uns an:

psychiat-praxis@thieme.de!

\section{Korrespondenzadresse}

Dr. med. Christa Roth-Sackenheim

Vorsitzende des Berufsverbands Deutscher Fachärzte für Psychiatrie und Psychotherapie, Mitglied des DGPPN-Vorstands

Nervenärztin in eigener Praxis

Breite Straße 63

56626 Andernach

C@Dr-Roth-Sackenheim.de

\section{Bibliografie}

Dol http://dx.doi.org/

$10.1055 / \mathrm{s}-0034-1387530$

Psychiat Prax 2015; 42: 66-67

(c) Georg Thieme Verlag KG

Stuttgart · New York

ISSN 0303-4259 